\title{
miR-200c Accelerates Hepatic Stellate Cell-Induced Liver Fibrosis via Targeting the FOG2/PI3K Pathway
}

\author{
Tengfei Ma, ${ }^{1,2,3}$ Xiuqin Cai, ${ }^{1,2,3}$ Zifeng Wang, ${ }^{2,3}$ Li Huang, ${ }^{4}$ Chang Wang, \\ Songshan Jiang, ${ }^{5}$ Yunpeng Hua, ${ }^{1}$ and Quentin Liu ${ }^{2,3}$ \\ ${ }^{1}$ Department of Liver Surgery, The First Affiliated Hospital, Sun Yat-sen University, Guangzhou 510080, China \\ ${ }^{2}$ Sun Yat-sen University Cancer Center, State Key Laboratory of Oncology in South China, Collaborative Innovation Center of \\ Cancer Medicine, Guangzhou, China \\ ${ }^{3}$ Institute of Cancer Stem Cell, Dalian Medical University, Dalian, China \\ ${ }^{4}$ Department of Biliary and Pancreatic Surgery, The First Affiliated Hospital, Sun Yat-sen University, Guangzhou 510080, China \\ ${ }^{5}$ School of Life Sciences, Sun Yat-sen University, Guangzhou 510080, China
}

Correspondence should be addressed to Yunpeng Hua; hyp0427@163.com and Quentin Liu; liuq9@mail.sysu.edu.cn

Received 5 February 2017; Accepted 21 March 2017; Published 13 June 2017

Academic Editor: Goutam Ghosh Choudhury

Copyright (C) 2017 Tengfei Ma et al. This is an open access article distributed under the Creative Commons Attribution License, which permits unrestricted use, distribution, and reproduction in any medium, provided the original work is properly cited.

Background. Although expression of miR-200s is aberrant in liver fibrosis, its role in liver fibrogenesis still remains unknown. Here, we investigated the role of miR-200c in the activation of human hepatic stellate cells (HSCs) and induction of liver fibrosis. Methods. We engineered human HSCs (LX2 cell line) to stably express miR-200c (LX2-200c) or empty vector control (LX2-nc). Results. miR-200c expression upregulated $\alpha$-smooth muscle actin (SMA) and vimentin, enhanced HSCs growth and migration, increased expression of collagen type I (a main component of ECM) gene and secretion of epidermal growth factor (EGF), and upregulated the phosphorylation of Akt, a downstream effector of the PI3K pathway. As a target of miR-200s and inhibitor of PI3K pathway, FOG2 protein expression was significantly suppressed in LX2-200c cells. Moreover, LY294002, a highly selective inhibitor of PI3K, blocked phosphorylation of Akt and the effects of miR-200c. Conclusions. These data suggest that miR-200c activates HSCs in liver fibrosis possibly by downregulating FOG2 protein expression and upregulating PI3K/Akt signaling. Autocrine activation of EGF signaling may also be a mechanism of miR-200c-mediated HSCs activation. So miR-200c can be a potential marker for HSCs activation and liver fibrosis progression, as well as a potential target to attenuate liver fibrosis.

\section{Introduction}

Liver fibrosis is a common outcome of chronic hepatic injuries including viral hepatitis, alcoholic or nonalcoholic steatohepatitis, autoimmune and chronic inflammatory conditions, and metabolic disorders. It usually progresses toward cirrhosis and eventually induces liver failure and hepatocellular carcinoma (HCC), which is recognized as a source of increasing morbidity and mortality worldwide. The prevalence of cirrhosis has been estimated at $0.3 \%$ in the United States and Western Europe and 1-2\% globally. It is the 14th leading cause of mortality globally, 12th leading cause of mortality in the United States, and the 4th leading cause in Central Europe and Mexico [1-3]. However, there are few effective therapies for liver fibrosis because its mechanisms are still complicated and poorly understood.

It is well-known that liver fibrosis is an excessive wound healing response to most forms of chronic liver disease and is characteristic of the excessive deposit of extracellular matrix (ECM) proteins. Hepatic stellate cells (HSCs) are recognized as the main matrix-producing cells in the liver. Following a fibrogenic stimulus, the HSCs change from quiescent vitamin A-storing cells to activated myofibroblast-like cells with ECM increasing dramatically, especially type I collagen. Besides secreting ECM, activated HSCs also produce a number of profibrotic cytokines and growth factors to both maintain and deteriorate the fibrotic process in paracrine and autocrine ways [4-9]. So HSCs may be a good target for prevention and treatment of liver fibrosis. 
TABLE 1: Primer sequences and/or ordering information used for qPCR analyses.

\begin{tabular}{lcc}
\hline Gene & \multicolumn{1}{c}{ Sense } & Antisense \\
\hline Collagen type I & $5^{\prime}$-GTGCGATGACGTGATCTGTGA-3' & 5'-TTGGTCGGTGGGTGACTCTG-3' $^{\prime}$ \\
GAPDH & $5^{\prime}$-GGAGCGAGATCCCTCCAAAAT-3' & $5^{\prime}$-GGCTGTTGTCATACTTCTCATGG-3' \\
Hsa-miR-200c & EXIQON, product number: 204482 & \\
U6 & EXIQON, product number: 203907 & \\
\hline
\end{tabular}

MicroRNAs (miRNAs) are endogenous small noncoding RNA sequences of 20-23 nucleotides that control gene expression by degrading target mRNAs or suppressing their translation. Over one-third of human genes appear to be the targets of conserved miRNAs, which are thought to be involved in various biological processes (normal growth and development) and pathological processes (cancer and other diseases). However, evidence is accumulating that miRNAs participate in the progression of liver fibrosis and regulation of HSCs proliferation/apoptosis [10-12]. The miR200s (miR-200a, miR-200b, miR-200c, miR-429, and miR141) have recently been implicated in tissue fibrosis [13, 14]. For example, expression of miR-200s was elevated in a mouse unilateral ureteral obstruction (UUO) model and the expression of miR-200a and -200b was increased in a mouse liver fibrosis model and human clinical samples. Feng et al. [15] also found upregulation of miR-200a, 200b, and $200 \mathrm{c}$ in fatty liver tissues. Moreover, upregulation of miR-200b stimulated proliferation and migration of HSCs via the PI3K/Akt signaling pathway [16], while upregulation of miR-200a inhibited TGF- $\beta 1$-induced HSC activation and proliferation [17]. However, few reports have addressed the role of miR-200c in HSC activation and liver fibrosis. Our results in the present study revealed that miR-200c plays a critical role in HSC activation and liver fibrosis progression via targeting FOG2 and activating PI3K/Akt signaling.

\section{Material and Methods}

2.1. Reagents and Materials. The human hepatic stellate cell line (LX-2) and the human embryonic kidney cell line cells (HEK 293T) were obtained from American Type Culture Collection (ATCC). Dulbecco's modified Eagle's medium (DMEM) was purchased from Invitrogen Life Technologies, Inc. (Carlsbad, CA, USA). Fetal bovine serum (FBS) and Opti-MEM were obtained from GIBCO (Los Angeles, CA, USA). Anti-Akt, anti-phospho-Akt, anti-GAPDH antibodies, and LY 294002 (PI3K inhibitor) were obtained from Cell Signaling Technology Inc. (Danvers, MA, USA). Anti-FOG2 and anti- $\alpha$-smooth muscle actin (SMA) antibodies were purchased from Abcam (New Territories, Hong Kong). Antivimentin antibody was obtained from Millipore (Schwalbach am Taunus, Germany). Lipofectamine 2000 and Trizol were obtained from Invitrogen. Cell Counting Kit-8 (CCK-8) was from Dojindo (Kumamoto, Japan). miExpress ${ }^{\mathrm{TM}}$ Precursor miRNA Expression (pEZX-MR03) clone was ordered from GeneCopoeia (Rockville, MD, USA). Universal-RTmicroRNA-PCR kits were from EXIQON (Vedbaek, Denmark). SYBR Green qPCR superMix was from Transgen Biotech (Beijing, China).
2.2. Cell Culture and Construction of the HSCs Stably Overexpressing miR-200c. The LX-2 cells and 293T cells were cultured in DMEM supplemented with $10 \%$ FBS, $100 \mathrm{U} / \mathrm{mL}$ penicillin, and $100 \mu \mathrm{g} / \mathrm{mL}$ streptomycin at $37^{\circ} \mathrm{C}$ in a humidified atmosphere of $5 \% \mathrm{CO}_{2}$. Briefly, we first generated miR200c-eGFP lentiviral particles or control scramble lentiviral particles. The $293 \mathrm{~T}$ cells were cotransfected with $5 \mu \mathrm{g}$ of either hsa-miR-200c-eGFP carrying plasmid (Catalog No.: HmiR0180-MR03, GeneCopoeia) or $5 \mu \mathrm{g}$ of pEZX-MR03 (Catalog No.: CmiR0001-MR03, GeneCopoeia) and second generation packaging plasmid (7.5 $\mu \mathrm{g}$ of psPAX2) and envelop plasmid $(2.5 \mu \mathrm{g}$ of $\mathrm{pMD} 2 \mathrm{G})$. The virus-containing supernatant was collected 48 and $72 \mathrm{~h}$ after transfection and passed through a $0.5 \mu \mathrm{m}$ sterile filter. Then, $2 \times 10^{4} \mathrm{LX} 2$ cells were infected in a $35 \mathrm{~mm}$ dish by $1 \mathrm{ml}$ of miR-200c-expressing or scramble lentiviruses containing $1 \mu \mathrm{l}$ of polybrene $(8 \mathrm{mg} / \mathrm{ml})$. Three days after transduction, the cells were trypsinized and replated in $60-\mathrm{mm}$ dishes. Puromycin $(2 \mu \mathrm{g} / \mathrm{ml})$ was added 3 days after transfection to select and purify the miR-200cexpressing or scramble clones.

2.3. Real Time qPCR. RNA was extracted using Trizol (Invitrogen) according to the manufacturer's instructions. cDNA was synthesized from $2 \mu \mathrm{g}$ of total RNA using a highcapacity cDNA Reverse Transcription Kit (Invitrogen) for mRNA and Universal cDNA synthesis kit II (EXIQON) for miRNA, respectively. Oligo dT primers were used for reverse transcription of mRNA, whereas stem-loop RT primers were employed for reverse transcription of miRNA. Real time quantitative PCR (qPCR) for miRNA expression analysis was performed on a LightCycler 480 Instrument II (Roche Life Sciences, Penzberg, Germany) using the Universal-RTmicroRNA-PCR Kit (EXIQON). qPCR for mRNA expression analysis was performed on an Bio-Rad CFX96 Real Time PCR System (Bio-Rad, Pleasanton, CA, USA) using the SYBR Green method (Invitrogen). GAPDH served as the control for mRNA analyses, and U6 snRNA served as the control for miRNA analyses. Primer sequences and/or ordering information used for qPCR analyses are displayed in Table 1. The relative expression levels of miRNAs and mRNAs were calculated by the 2-ddCt method.

2.4. Western Blot Analysis. Protein extraction and immunoblot analysis were performed as described previously [18]. Briefly, cells were washed three times with ice-cold PBS and lysed in radioimmunoprecipitation assay (RIPA) lysis buffer $(0.01 \mathrm{~mol} / \mathrm{L}$ sodium phosphate, $\mathrm{pH} 7.2,150 \mathrm{mmol} / \mathrm{L} \mathrm{NaCl}$, $2 \mathrm{mmol} / \mathrm{L}$ EDTA, 1\% NP-40, 1\% sodium deoxycholate, $0.1 \%$ SDS, $2 \mathrm{mmol} / \mathrm{L}$ AEBSF, $130 \mathrm{mmol} / \mathrm{L}$ bestatin, $14 \mathrm{mmol} / \mathrm{L} \mathrm{E}$ $64,0.3 \mathrm{mmol} / \mathrm{L}$ aprotinin, and $1 \mathrm{mmol} / \mathrm{L}$ leupeptin). Total cell 
lysates were resolved on denaturing and reducing 10-12\% SDS-PAGE, and the proteins were transferred from the gel onto Immobilon-P membranes. The membrane was blocked with $5 \%$ bovine serum albumin (BSA) in PBS, incubated with different antibodies, incubated with horseradish peroxidase(HRP-) conjugated secondary antibody, and treated with clarity enhanced chemiluminescence (ECL) substrate (Millipore) to visualize the protein bands.

2.5. Proliferative Analysis. LX2-200c and LX2-nc cells were seeded at $1 \times 10^{3}$ cells/well in 96-well plates, respectively, with or without treatment of LY294002, cultured for 0, 1, $2,3,4$, and 5 days, incubated with $10 \mu \mathrm{L}$ of CCK- 8 plus $100 \mu \mathrm{L}$ of DMEM/well for $2 \mathrm{~h}$, and then placed in a microplate reader (BioTek Synergy2, Winooski, VT, USA) to measure the absorbance at $450 \mathrm{~nm}$.

2.6. Wound Healing Assay. LX2-200c and LX2-nc cells were seeded at $5 \times 10^{4}$ cells/well in 6-well plates, respectively, with or without treatment of LY294002. When cells were grown to $80 \%$ confluence, a wound track was scraped using a sterile 200- $\mu$ l pipette tip to create a denuded zone of constant width in the middle of each well. Then, each well was washed with PBS twice to remove the detached cells. Cell migration to the wounded region was observed under a microscope. Images were captured at 0,12 , and $24 \mathrm{~h}$ to monitor the wound healing process. Migration ratio (\%) was calculated by dividing the width of the denuded zone at $12 \mathrm{~h}$ or $24 \mathrm{~h}$ by its width at $0 \mathrm{~h}$.

2.7. ELISA. The conditioned medium was collected. Briefly, LX2-200c and LX2-nc cells were seeded at $5 \times 10^{4}$ cells/well in 6-well plates, respectively, grown to $80 \%$ confluence, and washed with sterile PBS three times. The growth medium was replaced with an equal volume of serum-free DMEM and, after $24 \mathrm{~h}$, the supernatant was centrifuged to remove cells and particulates, collected, and stored at $-80^{\circ} \mathrm{C}$.

Human EGF protein levels were quantified by ELISA using the colorimetric sandwich ELISA kit (Proteintech, Chicago, ILUSA) according to the instruction manual. Briefly, samples and standards were diluted with PT 1-ec diluent in wells precoated with a monoclonal anti-human EGF antibody and treated with $1 \%$ BSA in PBS for $1 \mathrm{~h}$ to block nonspecific binding. The plates were incubated for $2 \mathrm{~h}$ at room temperature to allow capture of the EGF-tagged protein by the bound antibody, extensively washed with buffer, incubated with a biotinylated polyclonal antibody to $\mathrm{EGF}$ for $1 \mathrm{~h}$ at $37^{\circ} \mathrm{C}$ in a humidified environment, washed with buffer, incubated with Streptavidin-HRP for $40 \mathrm{~min}$, washed, incubated with TMB (tetramethylbenzidine) substrate for $10 \mathrm{~min}$, and incubated with stop solution to terminate the color reaction. Absorbance was measured at $450 \mathrm{~nm}$.

2.8. Statistical Analysis. Each experiment was performed in at least triplicate. Results are expressed as the mean \pm standard deviation. Data were analyzed using GraphPad Prism (GraphPad Software Inc., La Jolla, CA). Statistical analysis was performed using the Student $t$-test. $P<0.05$ was considered statistically significant.

\section{Results}

3.1. miR-200c Promotes the Activity, Proliferation, and Migration of HSCs. First, we constructed two cell lines from the human HSC line (LX2): one stably overexpressed miR-200c (LX2-200c) and its control that expressed empty vector (LX2nc) $(P=0.013$, Figures $1(\mathrm{a})$ and $1(\mathrm{~b}))$. We found that LX2200c had markedly higher levels of $\alpha$-SMA and vimentin (classical activation markers of HSCs [19]; Figure 1(c)) and significantly higher cell growth rate (all $P<0.05$; Figure $1(\mathrm{~d})$, and Table 1). The wound healing assay showed that more LX2200 c cells than LX-2-nc cells migrated into the wound track after $12 \mathrm{~h}$ and $24 \mathrm{~h}$. The wound track area after $12 \mathrm{~h}$ and $24 \mathrm{~h}$ was markedly smaller for LX2-200c than for LX2-nc $(50.7 \% \pm$ $3.1 \%$ and $23.7 \% \pm 2.5 \%$, respectively, versus $76.0 \% \pm 3.0 \%$ and $50.3 \% \pm 2.5 \%$, respectively; all $P<0.001$; Figures $1(\mathrm{e})$ and $1(\mathrm{f}))$.

3.2. miR-200c Activates the PI3K/Akt Pathway via Suppressing Expression of FOG2. Because activation of the PI3K/Akt pathway is required for HSC proliferation and migration [20], we further probed whether the proliferative and migratory effects of miR-200c are dependent on the activation of PI3K/Akt. Our results showed that the level of phosphorylation at Akt residue S473 was significantly higher in LX2200c cells than LX2-nc cells (Figure 2(a)). We also observed that $25 \mu \mathrm{M}$ LY-294002 (a PI3K inhibitor) significantly inhibited the expression of phospho-Akt in LX2-200c cells in a time-dependent manner (Figure 2(b)) and blocked the proliferation and migration of LX2-200c cells (Figures 2(c) and 2(d)). Moreover, the wound area for LX2-200c cells after $12 \mathrm{~h}$ and $24 \mathrm{~h}$ was significantly larger with LY-294002 treatment than without treatment $(79 \% \pm 3.0 \%$ and $57.7 \% \pm$ $3.5 \%$, respectively, versus $57.7 \% \pm 3.2 \%$ and $19.3 \% \pm 3.1 \%$, respectively; all $P<0.001$; Figures $2(\mathrm{~d})$ and $2(\mathrm{e})$ ).

To further probe the mechanism of miR-200c-mediated PI3K signaling, we measured the expression of FOG2 (a reported inhibitor of PI3K [16, 21-23]) and PI3K after miR-200c transfection and found that LX2-200c cells had significantly decreased levels of FOG2 while no significant change was observed in PI3K expression (Figure 2(f)), which is consistent with our previous finding of increased Akt phosphorylation.

3.3. miR-200c Overexpression Upregulates the Expression of Collagen Type I and Epidermal Growth Factor (EGF) via the FOG2/PI3K/Akt Pathway. Collagen type I is the main component of the ECM and dramatically increases in liver fibrosis [20]. We found that miR-200c overexpression significantly upregulated the gene expression of collagen type I in HSCs (Figure 3(a)). To assess whether PI3K/Akt signaling is involved in miR-200c-mediated collagen type I gene expression, we treated LX2-200c cells with $25 \mu \mathrm{M}$ LY294002 in the presence of $10 \%$ serum for $30 \mathrm{~min}$ and detected a marked drop in their collagen type I mRNA levels (Figure 3(b)).

Epidermal growth factor (EGF) plays a role in liver fibrosis through promoting the autocrine/paracrine proliferation and activation of HSCs [24-26]. Assessment of EGF protein level in the conditioned medium of HSCs revealed 

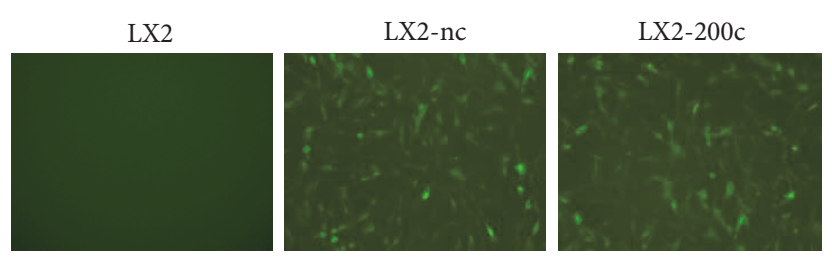

(a)

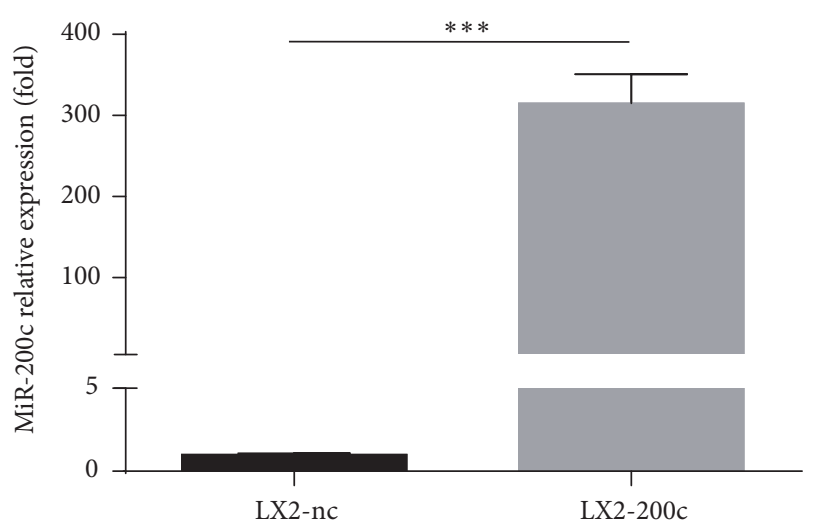

(b)

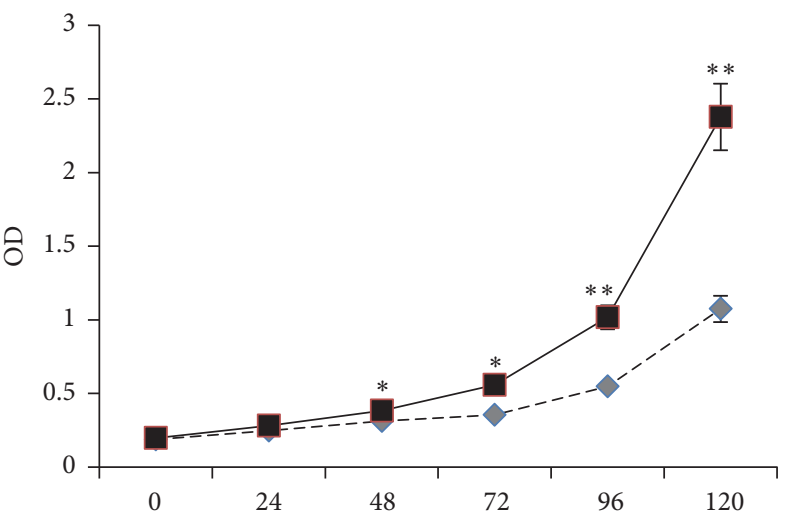

(h)

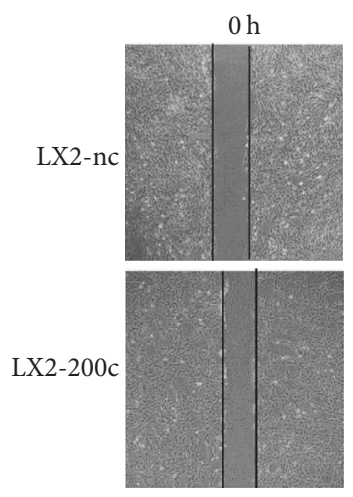

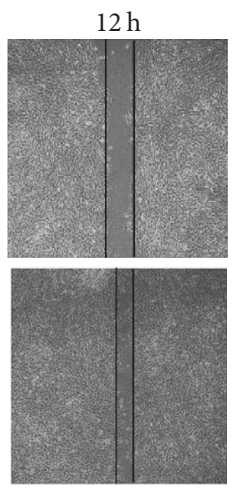

(e)

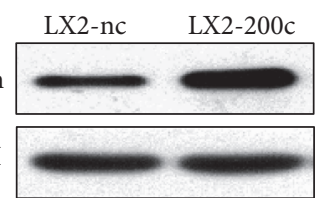

$24 \mathrm{~h}$
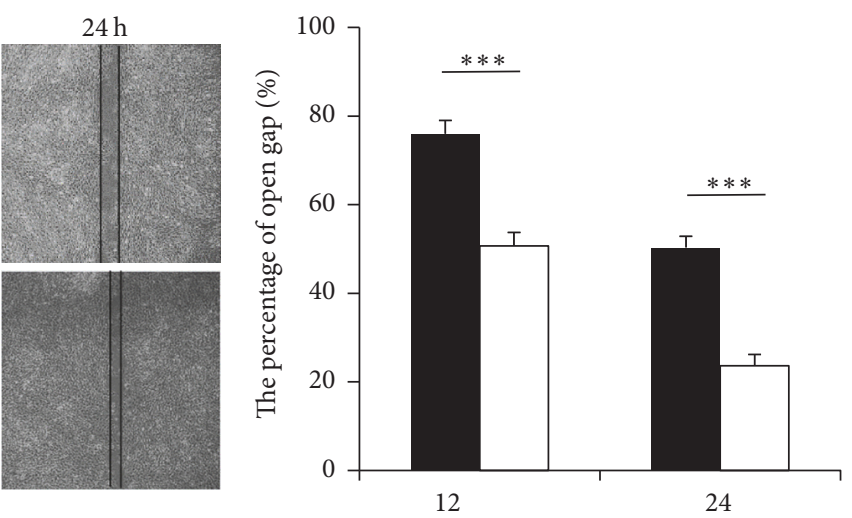

(h)

LX2-nc

$\square$ LX2-200c

(f)

FIGURE 1: miR-200c promotes the activity, proliferation, and migration of HSCs. (a, b) Construction of two cell lines (LX2-200c and LX2nc) from the human HSC line (LX2). LX2-200c stably overexpressed miR-200c and LX2-nc expressed empty vector as the control. Green fluorescent protein (GFP) expression was observed in LX2-200c and LX2-nc by fluorescence microscopy, rather than in LX2 (original magnification $\times 400$ ). The level of miR-200c expression in LX2-200c was approximately 314 times of that in LX2-nc cells. (c) LX2-200c had markedly higher levels of $\alpha$-SMA and vimentin. (d) miR-200c promoted the proliferation of LX2. (e, f) The migration of LX2 was increased by miR-200c. $*$ means $p<0.05, * *$ means $p<0.01$, and $* * *$ means $p<0.001$. 


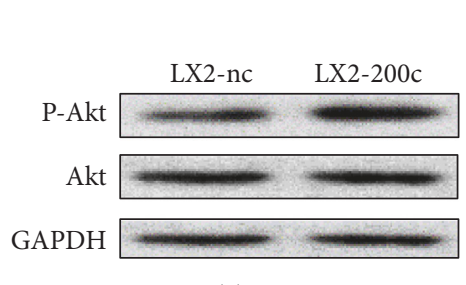

(a)

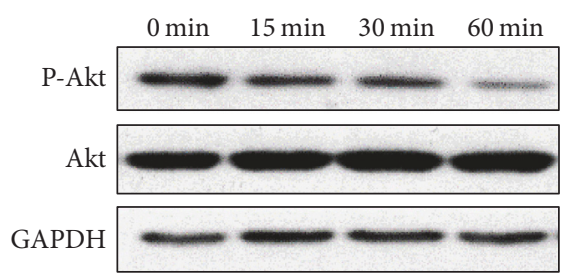

(b)

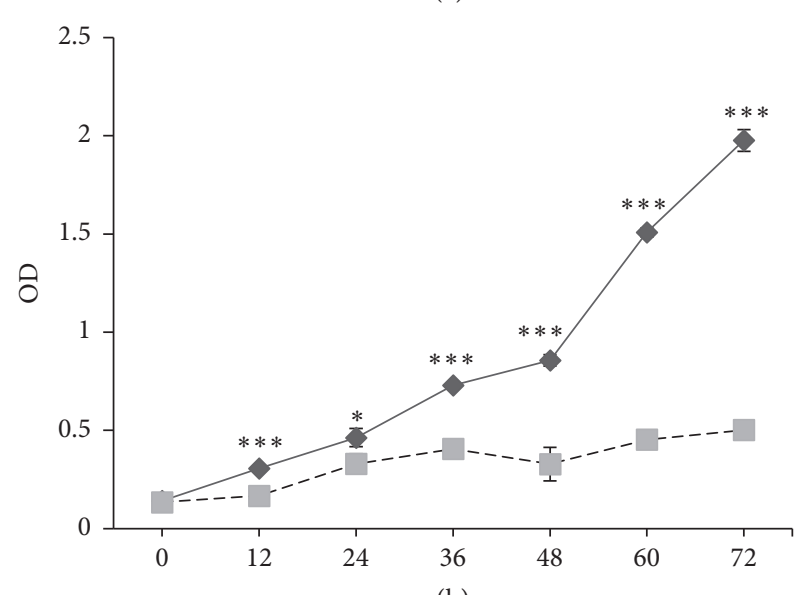

(h) (c)

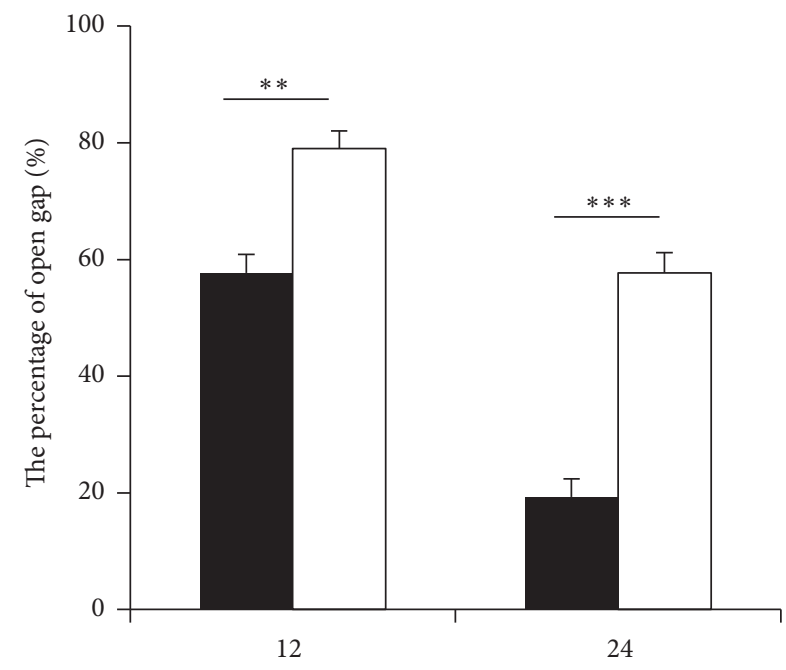

(h)

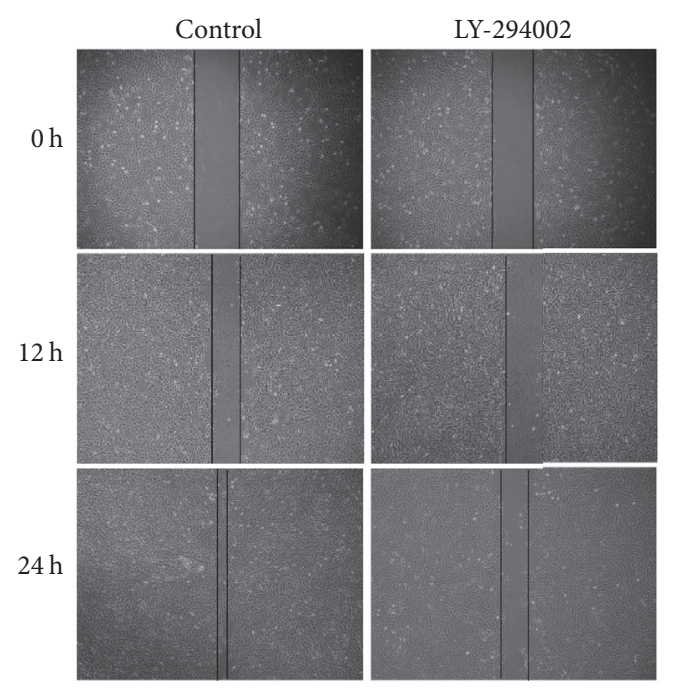

(d)

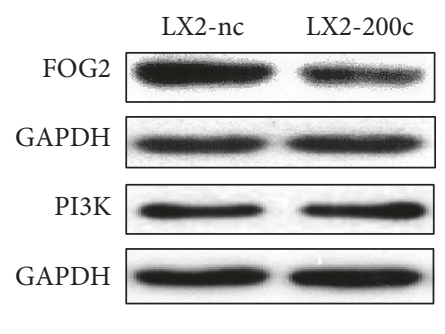

Control $\square$ LY-294002

(e)

(f)

FIGURE 2: miR-200c activates the PI3K/Akt pathway via suppressing expression of FOG2. (a) The level of phosphorylation at Akt residue S473 was significantly higher in LX2-200c cells than LX2-nc cells. (b) $25 \mu \mathrm{M}$ LY-294002 (a PI3K inhibitor) significantly inhibited the expression of phospho-Akt in LX2-200c cells in a time-dependent manner. (c-e) $25 \mu \mathrm{M}$ LY-294002 significantly inhibited the proliferation and migration of LX2-200c cells. (f) There was the significantly lower levels of FOG2 expression in LX2-200c cells, while no significant change was observed in $\mathrm{PI} 3 \mathrm{~K}$ expression. $*$ means $p<0.05, * *$ means $p<0.01$, and $* * *$ means $p<0.001$. 


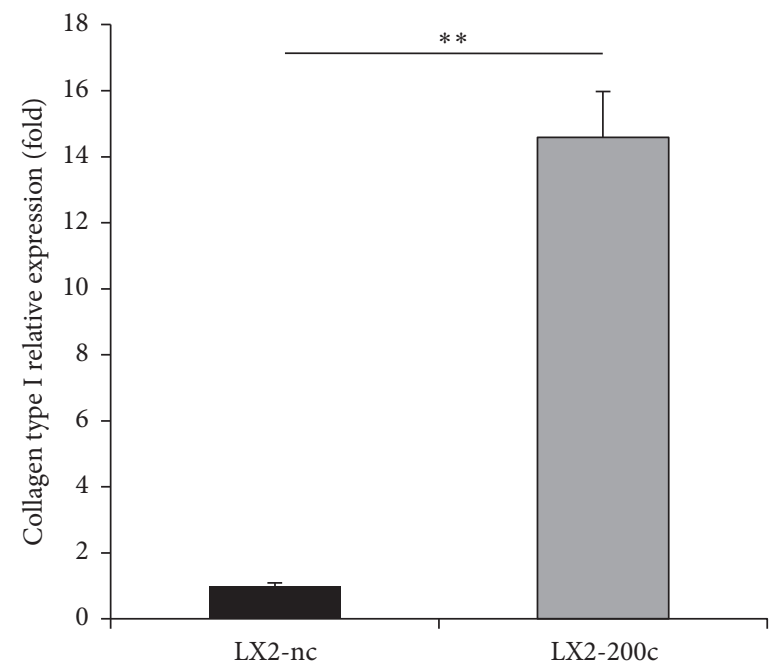

(a)

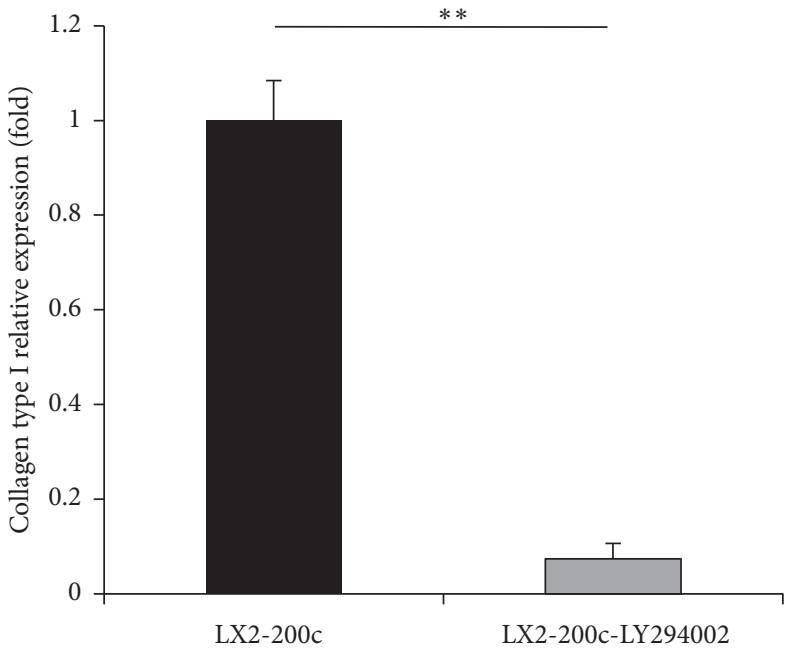

(b)

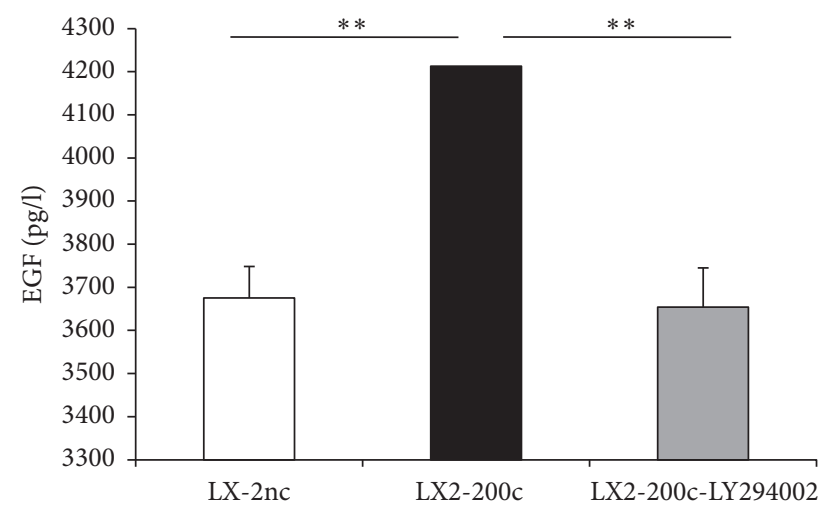

(c)

FIGURE 3: miR-200c overexpression upregulates the expression of collagen type I and epidermal growth factor (EGF) via the FOG2/PI3K/Akt pathway. (a, c) miR-200c overexpression significantly upregulated the gene expression of collagen type I and EGF secretion in HSCs. (b, c) $25 \mu \mathrm{M}$ LY-294002 (a PI3K inhibitor) significantly inhibited the gene expression of collagen type I and EGF secretion in LX2-200c cells. ** means $p<0.01$.

a significantly increased level of EGF secretion by HSCs after miR-200c transfection (Figure 3(c)), which could be inhibited by treatment with $25 \mu \mathrm{M}$ LY294002 for $30 \mathrm{~min}$ (Figure 3(c)). These findings proved that PI3K/Akt signaling is involved in miR-200c-enhanced secretion of EGF.

\section{Conclusion}

Hepatic stellate cells (HSCs) are thought to play a crucial role in liver fibrosis, as their activation following liver injury is responsible for increased synthesis and deposition of ECM proteins in the liver. In addition, the proliferation and migration of HSCs after activation effectively amplify the fibrotic response. Furthermore, profibrotic cytokines and growth factors secreted by activated HSCs (like transforming growth factor- $\beta$ [TGF- $\beta$ ], platelet-derived growth factor [PDGF], matrix metalloproteinases [MMPs], epidermal growth factor [EGF], leptin, and so on [4-9]) perpetuate the fibrotic process through paracrine and autocrine effects.

Although several studies have reported the involvement of miR-200s in the development of tissue fibrosis, including liver fibrosis, no study has implicated miR-200c. Additionally, miR-200a and miR-200b were found to have opposite effects on liver fibrosis development [13-17]. Herein, we showed that proliferation and migration of a human HSC (LX-2) cell line were enhanced by engineering it to stably overexpress miR200c (LX2-200c). The expressions of $\alpha$-SMA and vimentin (biomarkers of activated HSCs) and collagen type I (a major component of ECM) were found to be upregulated significantly in LX2-200c.

The phosphatidylinositol 3-kinase (PI3K)-Akt pathway plays a key role in cellular hypertrophy. The PI3K enzyme, a well-known upstream mediator of Akt kinase activation, is composed of a catalytic subunit, p110, and a regulatory subunit, p85 $\alpha$. The catalytic subunit of PI3K can recruit Akt kinase to the membrane and activate it by phosphorylation. Activated Akt further phosphorylates several downstream proteins that play central roles in hypertrophy, cell growth, cell survival, and protein synthesis [20]. However, a novel protein FOG2 binds to p $85 \alpha$, thereby inhibiting PI3K activation. Intriguingly, miR-200 was reported to decrease FOG2 
expression by targeting the $3^{\prime}$ UTR of the FOG2 mRNA, thereby altering PI3K activity and regulating the insulin signaling pathway and metabolism [16, 21]. Park et al. [22] indicated that FOG2 is downregulated by mimics of miR$200 \mathrm{~b}$ and miR-200c in mouse mesangial cells (MMC). Mei et al. [23] also found that miR-200c inhibited the expression of PTEN and FOG2 to promote the expansion and immune suppressive activity of myeloid-derived suppressor cells (MDSCs). In the present studies, we showed that transfection of miR-200c significantly reduced FOG2 protein levels in LX-2 cells, which subsequently led to PI3K/Akt signaling activation. To determine whether the effect of miR-200c is mediated through the FOG2/PI3K pathway, we used LY294002, a specific PI3 kinase inhibitor, to block PI3K activation in the LX-2 cells transfected with miR200c. The results showed that LY294002 treatment significantly inhibited miR-200c-enhanced LX-2 cell proliferation and migration and ECM deposition, which suggested that PI3K/Akt activation was essential to the profibrotic effect of miR-200c.

EGF plays a role in liver fibrosis, liver cirrhosis, and even hepatocellular carcinoma (HCC). EGF expression in the liver increases during cirrhosis, and the level of EGF mRNA expression is associated with poor survival of cirrhotic patients. Bachem et al. [24] indicated that EGF signaling triggered HSC proliferation in a receptor-dependent autocrine/paracrine manner. Zhang et al. [25] pointed out that EGF expression was significantly increased in activated HSCs, and EGF promotes HSC proliferation via activation of the EGF receptor (EGFR). Fuch et al. [26] reported that the small-molecule EGFR inhibitor erlotinib inhibited the activation of HSCs, prevented the progression of cirrhosis, and caused fibrosis to regress in some animal models. Our results showed that miR-200c overexpression also promoted the secretion of EGF in HSCs and that miR-200c-mediated EGF secretion could be inhibited by LY294002 treatment. However, our preliminary experiment showed that miR200 c overexpression had no effect on other growth factors, including transforming growth factor- $\beta 1$ (TGF- $\beta 1$ ), platelet-derived growth factor (PDGF), fibroblast growth factor (FGF), hepatocyte growth factor (HGF), and vascular endothelial growth factor (VEGF). So we speculated that miR-200c promoted HSC proliferation, migration, and ECM production in part via autocrine activation of EGF signaling, rather than signaling by other growth factors. In addition, PI3K/Akt signaling also played a key role in miR-200cmediated EGF secretion by HSCs.

In summary, our results show directly that miR-200c is an important promoter of HSCs activity, proliferation, and migration and has a very important role in liver fibrosis. We found that the effects of miR-200c on HSCs and liver fibrosis are mediated via downregulation of FOG2 protein synthesis, activation of PI3K/Akt signaling, upregulation of activation of autocrine EGF signaling, and upregulation of collagen type I production in HSCs (Figure 4). In view of its putative pathogenic role in HSCs, miR-200c may be a potential marker for HSC activation and liver fibrosis progression, and even a potential target for the treatment of liver fibrosis.

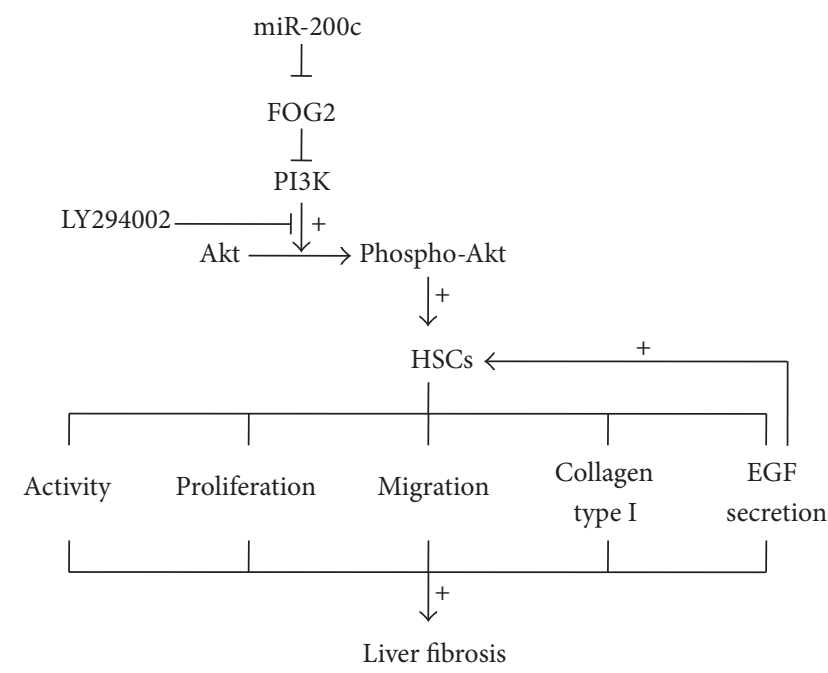

FIGURE 4: Schematic model of miR-200c accelerating hepatic stellate cell-induced liver fibrosis via targeting the FOG2/PI3K pathway. miR-200c activates HSCs and finally accelerates the progression of liver fibrosis via downregulation of FOG2 protein synthesis, activation of PI3K/Akt signaling. Autocrine activation of EGF signaling may also be a mechanism of miR-200c-mediated HSCs activation.

\section{Abbreviations}

HSCs: Hepatic stellate cells

SMA: $\quad \alpha$-Smooth muscle actin

EGF: Epidermal growth factor

HCC: Hepatocellular cancer

ECM: Extracellular matrix

miRNAs: MicroRNAs

UUO: Unilateral ureteral obstruction

DMEM: Dulbecco's modified Eagle's medium

FBS: $\quad$ Fetal bovine serum

qPCR: Real time quantitative PCR

RIPA: Radioimmunoprecipitation assay

BSA: Bovine serum albumin

ECL: $\quad$ Clarity enhanced chemiluminescence

TGF- $\beta$ : The transforming growth factor $\beta$

PDGF: Platelet-derived growth factor

MMPs: Matrix metalloproteinases

PI3K: Phosphatidylinositol 3-kinase

MMC: Mouse mesangial cells

MDSCs: Myeloid-derived suppressor cells

EGFR: EGF receptor

FGF: Fibroblast growth factor

HGF: Hepatocyte growth factor

VEGF: Vascular endothelial growth factor.

\section{Data Access}

The datasets during and/or analysed during the current study are available from the corresponding author on reasonable request.

\section{Conflicts of Interest}

The authors declare that they have no conflicts of interest. 


\section{Authors' Contributions}

Tengfei Ma and Xiuqin Cai contributed equally to this work. The project was conceived and directed by Yunpeng Hua and Quentin Liu. Data were collected, analyzed, and interpreted by Tengfei Ma, Xiuqin Cai, Zifeng Wang, Songshan Jiang, Chang Wang, and Yunpeng Hua. Statistical analysis was performed by Li Huang. The manuscript was written by Yunpeng Hua. All authors read and approved the final manuscript.

\section{Acknowledgments}

This study was supported by grants from the National Natural Science Foundation of China (no. 81201918), the Science and Technology Project of Guangdong Province (nos. 2012B031800099, 2014A020212626), the Doctorial Fellowship of Higher Education of China (no. 200805581172), and the Scientific Research Foundation for Returned Overseas Chinese Scholars and the State Education Ministry (no. 311, in 2015).

\section{References}

[1] V. Hernandez-Gea and S. L. Friedman, "Pathogenesis of liver fibrosis," Annual Review of Pathology, vol. 6, pp. 425-456, 2011.

[2] E. A. Tsochatzis, J. Bosch, and A. K. Burroughs, "Liver cirrhosis," The Lancet, vol. 383, no. 9930, pp. 1749-1761, 2014.

[3] O. Ramos-Lopez, E. Martinez-Lopez, S. Roman, N. A. Fierro, and A. Panduro, "Genetic, metabolic and environmental factors involved in the development of liver cirrhosis in Mexico," World Journal of Gastroenterology, vol. 21, no. 41, pp. 11552-11566, 2015.

[4] Z. Liu, J. Yi, R. Ye et al., "miR-144 regulates transforming growth factor- $\beta 1$ iduced hepatic stellate cell activation in human fibrotic liver," International Journal of Clinical and Experimental Pathology, vol. 8, pp. 3994-4000, 2015.

[5] N. Ikeda, S. Murata, T. Maruyama et al., "Platelet-derived adenosine 5'-triphosphate suppresses activation of human hepatic stellate cell: In vitro study," Hepatology Research, vol. 42, no. 1, pp. 91-102, 2012.

[6] R. Bansal, J. van Baarlen, G. Storm, and J. Prakash, “The interplay of the Notch signaling in hepatic stellate cells and macrophages determines the fate of liver fibrogenesis," Scientific Reports, vol. 5, Article ID 18272, 2015.

[7] M. Mikula, V. Proell, A. N. M. Fischer, and W. Mikulits, "Activated hepatic stellate cells induce tumor progression of neoplastic hepatocytes in a TGF- $\beta$ dependent fashion," Journal of Cellular Physiology, vol. 209, no. 2, pp. 560-567, 2006.

[8] K. Taura, S. de Minicis, E. Seki et al., "Hepatic stellate cells secrete angiopoietin 1 that induces angiogenesis in liver fibrosis," Gastroenterology, vol. 135, no. 5, pp. 1729-1738, 2008.

[9] B.-B. Wang, J.-Y. Cheng, H.-H. Gao, Y. Zhang, Z.-N. Chen, and H. Bian, "Hepatic stellate cells in inflammation-fibrosiscarcinoma axis," Anatomical Record, vol. 293, no. 9, pp. 14921496, 2010.

[10] J. Lambrecht, I. Mannaerts, and L. A. van Grunsven, "The role of miRNAs in stress-responsive hepatic stellate cells during liver fibrosis," Frontiers in Physiology, vol. 6, article 209, 2015.
[11] J. Du, X. Niu, Y. Wang et al., "MiR-146a-5p suppresses activation and proliferation of hepatic stellate cells in nonalcoholic fibrosing steatohepatitis through directly targeting Wnt1 and Wnt5a," Scientific Reports, vol. 5, Article ID 16163, 2015.

[12] M. Coll, A. E. Taghdouini, L. Perea et al., "Integrative miRNA and gene expression profiling analysis of human quiescent hepatic stellate cells," Scientific Reports, vol. 5, Article ID 11549, 2015.

[13] S. Yang, S. Banerjee, A. de Freitas et al., "Participation of miR200 in pulmonary fibrosis," The American Journal of Pathology, vol. 180, no. 2, pp. 484-493, 2012.

[14] Y. Murakami, H. Toyoda, M. Tanaka et al., "The progression of liver fibrosis is related with overexpression of the miR-199 and 200 families," PLoS ONE, vol. 6, no. 1, Article ID e16081, 2011.

[15] Y. Y. Feng, X. Q. Xu, C. B. Ji, C. M. Shi, X. R. Guo, and J. F. Fu, "Aberrant hepatic MicroRNA expression in nonalcoholic fatty liver disease," Cellular Physiology and Biochemistry, vol. 34, no. 6, pp. 1983-1997, 2014.

[16] Y. Xiao, J. Wang, Y. Chen et al., "Up-regulation of miR-200b in biliary atresia patients accelerates proliferation and migration of hepatic stallate cells by activating PI3K/Akt signaling," Cellular Signalling, vol. 26, no. 5, pp. 925-932, 2014.

[17] X. Sun, Y. He, T.-T. Ma, C. Huang, L. Zhang, and J. Li, "Participation of miR-200a in TGF- $\beta 1$-mediated hepatic stellate cell activation," Molecular and Cellular Biochemistry, vol. 388, no. 1-2, pp. 11-23, 2014.

[18] Y. Hua, S. White-Gilbertson, J. Kellner et al., "Molecular chaperone gp96 is a novel therapeutic target of multiple myeloma," Clinical Cancer Research, vol. 19, no. 22, pp. 6242-6251, 2013.

[19] J. Kim, S. Wang, J. Hyun et al., "Hepatic stellate cells express thymosin beta 4 in chronically damaged liver," PLOS ONE, vol. 10, no. 3, Article ID e0122758, 2015.

[20] S. Reif, A. Lang, J. N. Lindquist et al., "The role of focal adhesion kinase-phosphatidylinositol 3-kinase-Akt signaling in hepatic stellate cell proliferation and type I collagen expression," Journal of Biological Chemistry, vol. 278, no. 10, pp. 8083-8090, 2003.

[21] S. Hyun, J. H. Lee, H. Jin et al., "Conserved MicroRNA miR-8/miR-200 and its target USH/FOG2 control growth by regulating PI3K," Cell, vol. 139, no. 6, pp. 1096-1108, 2009.

[22] J. T. Park, M. Kato, H. Yuan et al., "FOG2 protein downregulation by transforming growth factor- $\beta 1$-induced MicroRNA-200b/c leads to akt kinase activation and glomerular mesangial hypertrophy related to diabetic nephropathy," The Journal of Biological Chemistry, vol. 288, no. 31, pp. 22469-22480, 2013.

[23] S. Mei, J. Xin, Y. Liu et al., "MicroRNA-200c promotes suppressive potential of myeloid-derived suppressor cells by modulating PTEN and FOG2 expression," PLoS ONE, vol. 10, no. 8, Article ID e0135867, 2015.

[24] M. G. Bachem, U. Riess, and A. M. Gressner, "Liver fat storing cell proliferation is stimulated by epidermal growth factor/transforming growth factor alpha and inhibited by transforming growth factor beta," Biochemical and Biophysical Research Communications, vol. 162, no. 2, pp. 708-714, 1989.

[25] D. Zhang, J. Zhang, X. Jiang et al., "Heparin-binding epidermal growth factor-like growth factor: a hepatic stellate cell proliferation inducer via ErbB receptors," Journal of Gastroenterology and Hepatology, vol. 29, no. 3, pp. 623-632, 2014.

[26] B. C. Fuchs, Y. Hoshida, T. Fujii et al., "Epidermal growth factor receptor inhibition attenuates liver fibrosis and development of hepatocellular carcinoma," Hepatology, vol. 59, no. 4, pp. 15771590, 2014. 


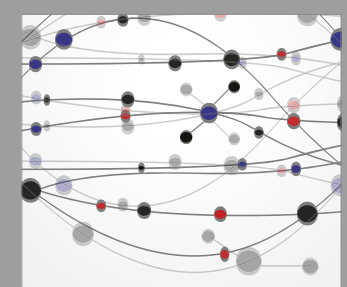

The Scientific World Journal
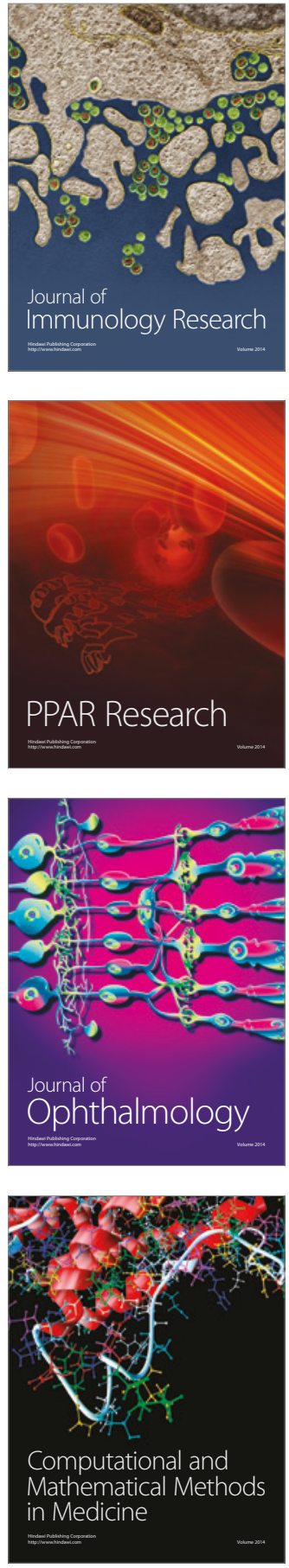

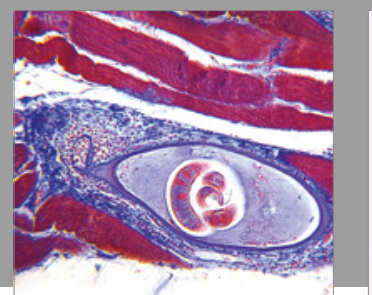

Gastroenterology Research and Practice
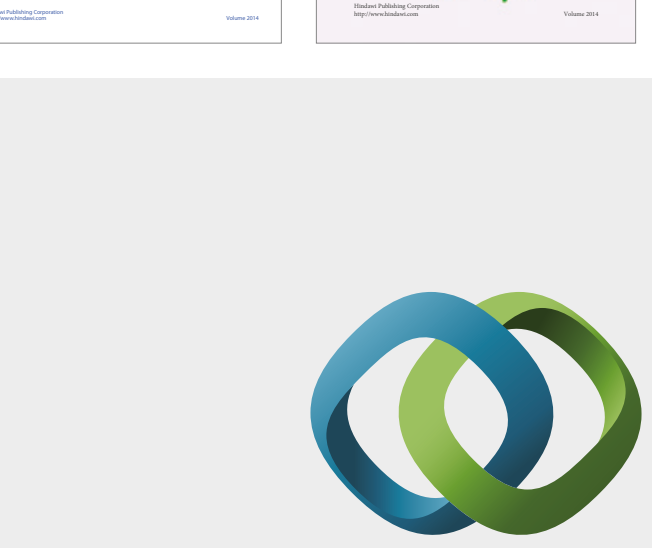

\section{Hindawi}

Submit your manuscripts at

https://www.hindawi.com
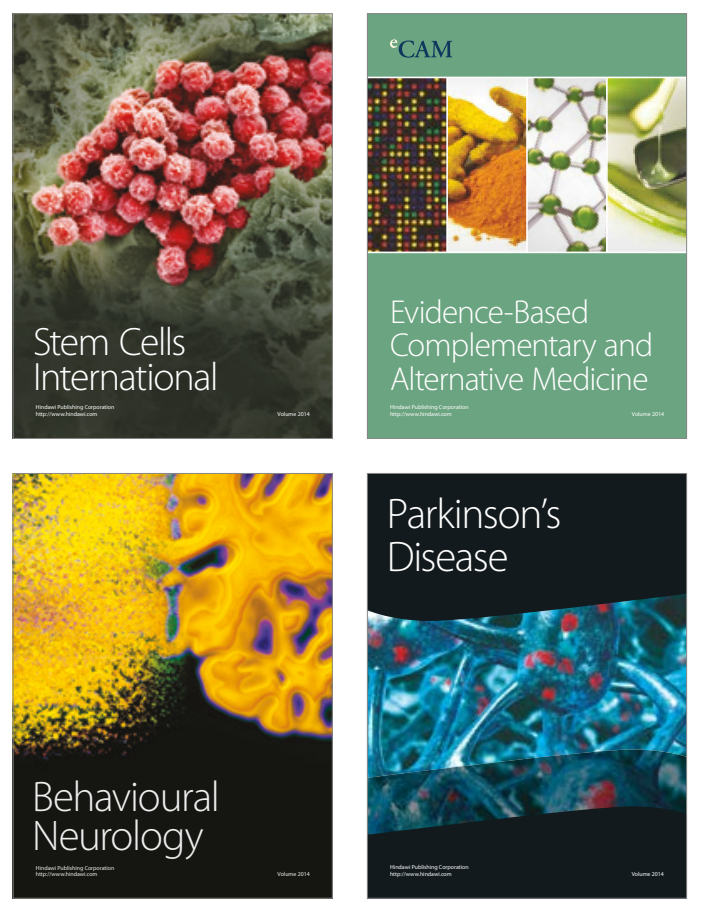
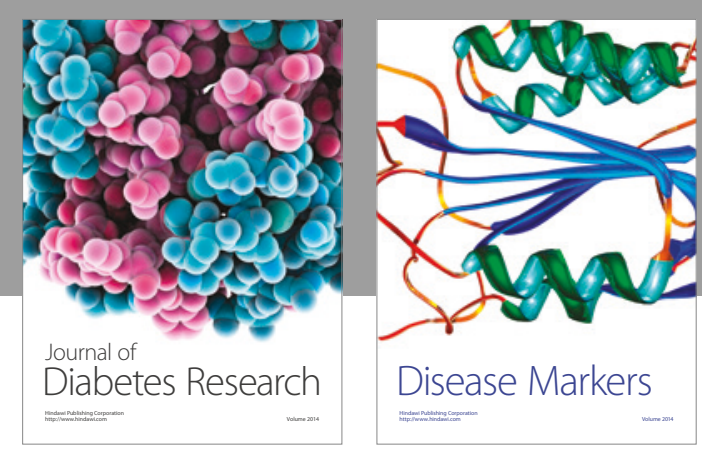

Disease Markers
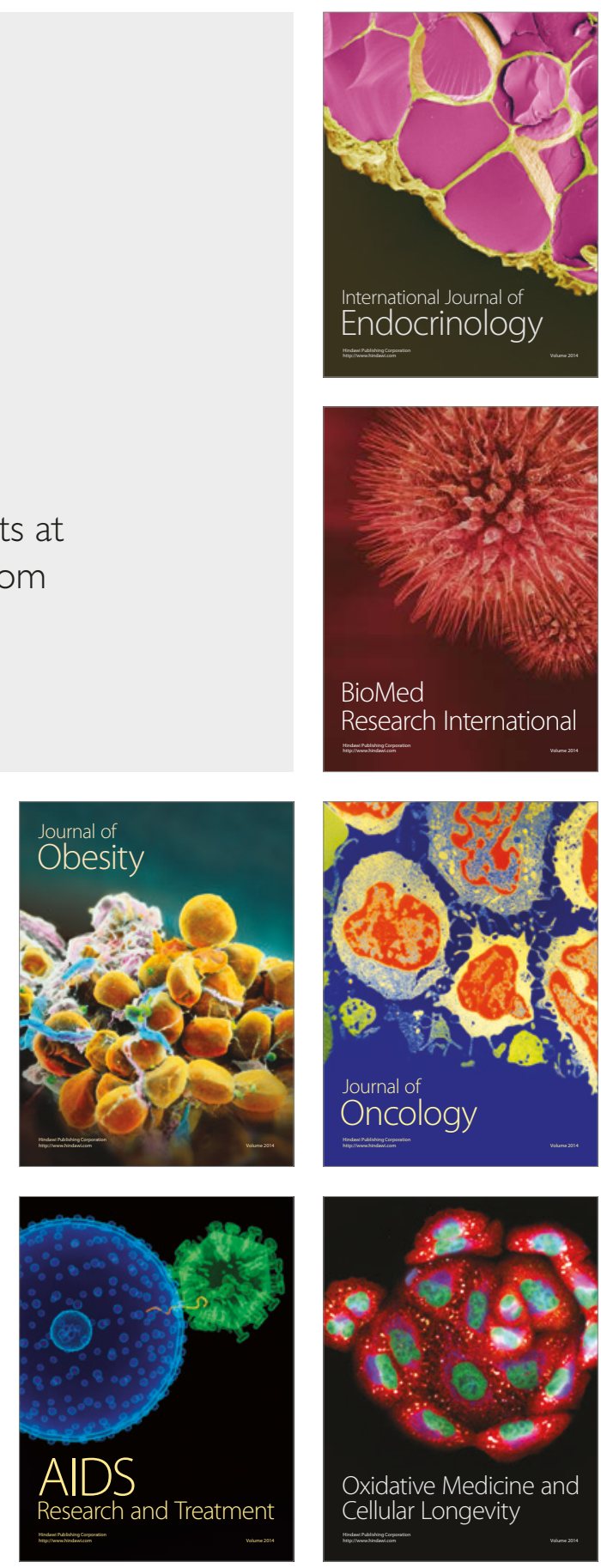